\title{
ENDPOINT STRICHARTZ ESTIMATES
}

\author{
By Markus Keel and Terence Tao
}

\begin{abstract}
We prove an abstract Strichartz estimate, which implies previously unknown endpoint Strichartz estimates for the wave equation (in dimension $n \geq 4$ ) and the Schrödinger equation (in dimension $n \geq 3$ ). Three other applications are discussed: local existence for a nonlinear wave equation; and Strichartz-type estimates for more general dispersive equations and for the kinetic transport equation.
\end{abstract}

1. Introduction. In this paper we shall prove a Strichartz estimate in the following abstract setting (see below for the concrete examples of the wave and Schrödinger equation): let $(X, d x)$ be a measure space and $H$ a Hilbert space. We'll write the Lebesgue norm of a function $f: X \rightarrow \mathbf{C}$ by

$$
\|f\|_{p} \equiv\|f\|_{L^{p}(X)} \equiv\left(\int_{X}|f(x)|^{p} d x\right)^{\frac{1}{p}}
$$

Suppose that for each time $t \in \mathbf{R}$ we have an operator $U(t): H \rightarrow L^{2}(X)$ which obeys the energy estimate:

- For all $t$ and all $f \in H$ we have

$$
\|U(t) f\|_{L_{x}^{2}} \lesssim\|f\|_{H}
$$

and that for some $\sigma>0$, one of the following decay estimates:

- For all $t \neq s$ and all $g \in L^{1}(X)$

$$
\left\|U(s)(U(t))^{*} g\right\|_{\infty} \lesssim|t-s|^{-\sigma}\|g\|_{1} \quad \text { (untruncated decay) }
$$

- For all $t, s$ and $g \in L^{1}(X)$

$$
\left\|U(s)(U(t))^{*} g\right\|_{\infty} \lesssim(1+|t-s|)^{-\sigma}\|g\|_{1} \quad \text { (truncated decay) }
$$

We will completely ignore any issues concerning whether $(U(t))^{*}$ are defined on

Manuscript received July 25, 1997.

Research of the second author supported in part by an NSF grant.

American Journal of Mathematics 120 (1998), 955-980. 
all of $L_{x}^{1}$ or only on a dense subspace. Our goal is to determine which space-time norms

$$
\|F\|_{L_{t}^{q} L_{x}^{r}} \equiv\left(\int\|F(t)\|_{L_{x}^{r}}^{q} d t\right)^{\frac{1}{q}}
$$

are controlled by (1),(2) or (1),(3). Remark that in the P.D.E. settings of the wave and Schrödinger equations we will set $\sigma=\frac{n-1}{2}, \frac{n}{2}$, respectively, $X=\mathbf{R}^{n}$, and $H=L^{2}\left(\mathbf{R}^{n}\right)$.

Definition 1.1. We say that the exponent pair $(q, r)$ is $\sigma$-admissible if $q, r \geq 2$, $(q, r, \sigma) \neq(2, \infty, 1)$ and

$$
\frac{1}{q}+\frac{\sigma}{r} \leq \frac{\sigma}{2}
$$

If equality holds in (4) we say that $(q, r)$ is sharp $\sigma$-admissible, otherwise we say that $(q, r)$ is nonsharp $\sigma$-admissible. Note in particular that when $\sigma>1$ the endpoint

$$
P=\left(2, \frac{2 \sigma}{\sigma-1}\right)
$$

is sharp $\sigma$-admissible.

THEOREM 1.2. If $U(t)$ obeys (1) and (2), then the estimates

$$
\begin{gathered}
\left\|\int(U(s))^{*} F(s) d s\right\|_{H} \lesssim\|F\|_{L_{t}^{q^{\prime}} L_{x}^{r^{\prime}}}, \\
\left\|\int_{s<t} U(t)(U(s))^{*} F(s) d s\right\|_{L_{t}^{q} L_{x}^{r}} \lesssim\|F\|_{L_{t}^{\tilde{q}^{\prime}} L_{x}^{\tilde{r}^{\prime}}}
\end{gathered}
$$

hold for all sharp $\sigma$-admissible exponent pairs $(q, r),(\tilde{q}, \tilde{r})$. Furthermore, if the decay hypothesis is strengthened to (3), then (5), (6) and (7) hold for all $\sigma$ admissible $(q, r)$ and $(\tilde{q}, \tilde{r})$.

As a consequence of Theorem 1.2 we can prove the endpoint Strichartz estimates for the wave and Schrödinger equation in higher dimensions. This completely settles the problem of determining the possible homogeneous Strichartz estimates for the wave and Schrödinger equations in higher dimensions. (The problem of determining all the possible retarded Strichartz estimates is still open.) For a given dimension $n$, we say that a pair $(q, r)$ of exponents is wave-admissible 


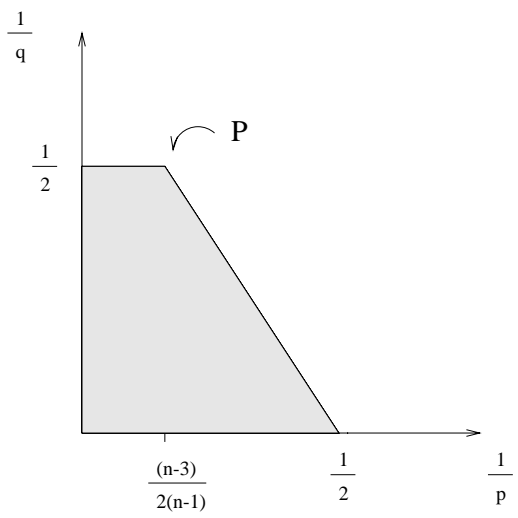

Figure 1. For $n>3$, the closed region is wave-admissible.

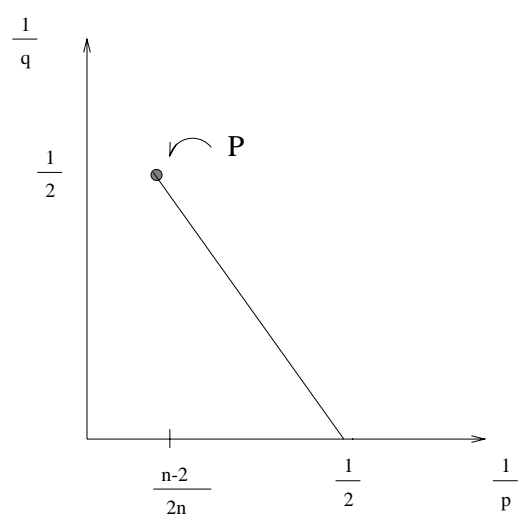

Figure 2. For $n>2$, the closed line segment is Schrödinger-admissible.

if $n \geq 2$ and $(q, r)$ is $\frac{n-1}{2}$-admissible, and Schrödinger-admissible if $n \geq 1$ and $(q, r)$ is sharp $\frac{n}{2}$-admissible. In particular, $P=\left(2, \frac{2(n-1)}{n-3}\right)$ is wave-admissible for $n>3$ (see Figure 1), and $P=\left(2, \frac{2 n}{n-2}\right)$ is Schrödinger-admissible for $n>2$ (see Figure 2).

In the following, we use $\dot{H}^{\gamma}=(\sqrt{-\Delta})^{-\gamma} L^{2}\left(\mathbf{R}^{n}\right)$ to denote the homogeneous Sobolev space.

COROllary 1.3. Suppose that $n \geq 2$ and $(q, r)$ and $(\tilde{q}, \tilde{r})$ are wave-admissible pairs with $r, \tilde{r}<\infty$. If $u$ is a (weak) solution to the problem

$$
\left\{\begin{array}{l}
\left(-\frac{\partial^{2}}{\partial t^{2}}+\Delta\right) u(t, x)=F(t, x), \quad(t, x) \in[0, T] \times \mathbf{R}^{n} \\
u(0, \cdot)=f, \partial_{t} u(0, \cdot)=g
\end{array}\right.
$$


for some data $f, g, F$ and time $0<T<\infty$, then

$$
\begin{gathered}
\|u\|_{L^{q}\left([0, T] ; L_{x}^{r}\right)}+\|u\|_{C\left([0, T] ; \dot{H}^{\gamma}\right)}+\left\|\partial_{t} u\right\|_{C\left([0, T] ; \dot{H}^{\gamma-1}\right)} \\
\lesssim\|f\|_{\dot{H}^{\gamma}}+\|g\|_{\dot{H}^{\gamma-1}}+\|F\|_{L^{\tilde{q}^{\prime}\left([0, T] ; L_{x}^{\tilde{r}^{\prime}}\right)}}
\end{gathered}
$$

under the assumption that the dimensional analysis (or "gap") condition

$$
\frac{1}{q}+\frac{n}{r}=\frac{n}{2}-\gamma=\frac{1}{\tilde{q}^{\prime}}+\frac{n}{\tilde{r}^{\prime}}-2
$$

holds. Conversely, if (9) holds for all $f, g, F, T$, then $(q, r)$ and $(\tilde{q}, \tilde{r})$ must be waveadmissible and the gap condition must hold.

When $r=\infty$ the estimate (9) holds with the $L_{x}^{r}$ norm replaced with the Besov norm $\dot{B}_{r, 2}^{0}$, and similarly for $\tilde{r}=\infty$.

COROLlaRy 1.4. Suppose that $n \geq 1$ and $(q, r)$ and $(\tilde{q}, \tilde{r})$ are Schrödingeradmissible pairs. If $u$ is a (weak) solution to the problem

$$
\left\{\begin{array}{l}
\left(i \frac{\partial}{\partial t}+\Delta\right) u(t, x)=F(t, x), \quad(t, x) \in[0, T] \times \mathbf{R}^{n} \\
u(0, \cdot)=f
\end{array}\right.
$$

for some data $f, F$ and time $0<T<\infty$, then

$$
\|u\|_{L_{t}^{q}\left([0, T] ; L_{x}^{r}\right)}+\|u\|_{C\left([0, T] ; L^{2}\right)} \lesssim\|f\|_{L^{2}}+\|F\|_{L^{\tilde{q}^{\prime}\left([0, T] ; L_{x}^{\tilde{r}^{\prime}}\right.}} \cdot
$$

Conversely, if the above estimate holds for all $f, F, T$, then $(q, r)$ and $(\tilde{q}, \tilde{r})$ must be Schrödinger-admissible.

Here we are using the convention that $\|u\|_{C([0, T] ; X)}=\infty$ when $u \notin C([0, T] ; X)$; thus Corollary 1.3 asserts that $u(t)$ is both bounded and continuous in $t$ in the space $\dot{H}^{\gamma}$, and similarly for Corollary 1.4 .

We have not stated the most general form of the estimates (9), (11): fractional differentiation, Sobolev imbedding, and Hölder's inequality all provide ways to modify the statements, and the case $T=\infty$ can be handled by the usual limiting argument. The gap condition (10), which was dictated by dimensional analysis, can be removed if one places an appropriate number of derivatives on the various terms in (9).

In the case when $\sigma>1$ and $(q, r)$ or $(\tilde{q}, \tilde{r})$ take the endpoint value $P$, the content of Corollaries 1.3-1.4 is new. These results extend a long line of investigation going back to a specific space-time estimate for the linear Klein-Gordon equation in [18] and the fundamental paper of Strichartz [24] drawing the connection to the restriction theorems of Tomas and Stein. For proofs of previously 


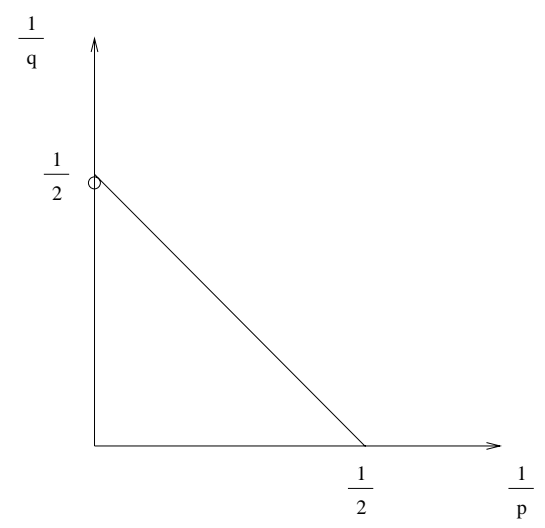

Figure 3. In $\mathbf{R}^{3+1}$, the wave equation estimate (8) with $q=2, r=\infty$ is known to be false. The Schrödinger estimate (11) in $\mathbf{R}^{2+1}$ with the same Lebesgue pair is also false.

known Strichartz-type wave equation estimates, see [14], [9], [15] and especially the careful expositions in [7], [21], [5]. For Strichartz-type results for the Schrödinger equation, see [6], [27].

When $\sigma=1$ the endpoint $P$ is inadmissable and the estimate for the wave equation $(n=3)$ and Schrödinger equation $(n=2)$ are known to be false [12], [16]. The problem of finding a satisfactory substitute for this estimate is still open.

There are several advantages to formulating Theorem 1.2 in this level of generality. First, it allows both wave equation and Schrödinger equation estimates to be treated in a unified manner. Second, it eliminates certain distractions and unnecessary assumptions (e.g. group structure on the $U(t)$ ). Finally, there is a natural scaling to these estimates which is only apparent in this setting. More precisely, the sharp statement of the theorem is invariant under the scaling

$$
\begin{gathered}
U(t) \leftarrow U\left(\frac{t}{\lambda}\right), \quad(U(s))^{*} \leftarrow\left(U\left(\frac{s}{\lambda}\right)\right)^{*}, \\
d x \leftarrow \lambda^{\sigma} d x, \quad\langle f, g\rangle_{H} \leftarrow \lambda^{\sigma}\langle f, g\rangle_{H} .
\end{gathered}
$$

In other words, for scaling purposes time behaves like $\mathbf{R}, X$ behaves like $\mathbf{R}^{\sigma}, H$ behaves like $L^{2}\left(\mathbf{R}^{\sigma}\right)$, and $U(t)$ is dimensionless. In practice the scaling dimension $\sigma$ differs from the Euclidean dimension; for instance, in the wave equation $\sigma=$ $(n-1) / 2$, and in the Schrödinger equation $\sigma=n / 2$.

Acknowledgments. The authors thank Sergiu Klainerman and Luis Vega for introducing them to this problem, Chris Sogge and Tom Wolff for sharing a number of very useful insights about the wave equation, and Elias Stein and Carlos Kenig for their helpful comments and encouragement. 
2. Outline of paper. Theorem 1.2 will be proved in several stages. In Section 3 we prove the homogeneous estimate (5) and its adjoint (6) away from the endpoint $P$ using the usual techniques of the $T T^{*}$ method and interpolation between the energy estimate and the decay estimate. The proof of the endpoint homogeneous estimate in Sections 4-6 requires a refined version of this argument; ironically, the estimate will be obtained by a bilinear interpolation between the nonendpoint results, together with the decay and energy estimates. We give two proofs of the bilinear interpolation step: a concrete one using an explicit decomposition of the functions involved (Section 5), and an abstract argument appealing to real interpolation theory (Section 6).

Finally, we have to modify the arguments for the homogeneous case to treat the retarded estimate (7). The most critical cases of the retarded estimate can be obtained directly from the corresponding homogeneous estimates, and the rest can be proved by interpolation and suitable variations of the homogeneous arguments. Curiously, our methods will be able to show (7) for certain exponents $(q, r),(\tilde{q}, \tilde{r})$ which are not both $\sigma$-admissible.

In the above arguments, we view the results as bilinear form estimates rather than operator estimates. The symmetry and flexibility of this viewpoint will be exploited heavily in the proof of the endpoint estimate.

In Section 8 we prove Corollaries 1.3 and 1.4. The argument follows standard techniques (see [7], [14], [21], [5] for the wave equation, and [6], [27] for the Schrödinger equation); the main difference is that the usual Strichartz interpolation method is replaced by Theorem 1.2.

In Section 9 we present an application of this endpoint inequality, obtaining an endpoint version of the well-posedness results of [10],[14] for the semilinear wave equation. In the final section we generalize Theorem 1.2 and discuss some applications to other problems, such as the kinetic transport equation and general dispersive equations.

3. The nonendpoint homogeneous estimate. In this section we prove the estimates (5), (6) when $(q, r) \neq P$.

By duality, (5) is equivalent to (6). By the $T T^{*}$ method, (6) is in turn equivalent to the bilinear form estimate

$$
\left|\iint\left\langle(U(s))^{*} F(s),(U(t))^{*} G(t)\right\rangle d s d t\right| \lesssim\|F\|_{L_{t}^{q^{\prime}} L_{x}^{r^{\prime}}}\|G\|_{L_{t}^{q^{\prime}} L_{x}^{r^{\prime}}}
$$

By symmetry it suffices to restrict our attention to the retarded version of (13),

$$
|T(F, G)| \lesssim\|F\|_{L_{t}^{q^{\prime}} L_{x}^{r^{\prime}}}\|G\|_{L_{t}^{q^{\prime}} L_{x}^{r^{\prime}}}
$$


where $T(F, G)$ is the bilinear form

$$
T(F, G)=\iint_{s<t}\left\langle(U(s))^{*} F(s),(U(t))^{*} G(t)\right\rangle d s d t
$$

By (real) interpolation between the bilinear form of (1)

$$
\left|\left\langle(U(s))^{*} F(s),(U(t))^{*} G(t)\right\rangle\right| \lesssim\|F(s)\|_{2}\|G(t)\|_{2}
$$

and the bilinear form of (2)

$$
\left|\left\langle(U(s))^{*} F(s),(U(t))^{*} G(t)\right\rangle\right| \lesssim|t-s|^{-\sigma}\|F(s)\|_{1}\|G(t)\|_{1}
$$

we obtain

$$
\left|\left\langle(U(s))^{*} F(s),(U(t))^{*} G(t)\right\rangle\right| \lesssim|t-s|^{-1-\beta(r, r)}\|F(s)\|_{r^{\prime}}\|G(t)\|_{r^{\prime}}
$$

where $\beta(r, \tilde{r})$ is given by

$$
\beta(r, \tilde{r})=\sigma-1-\frac{\sigma}{r}-\frac{\sigma}{\tilde{r}}
$$

Using (4), one checks that $\beta(r, r) \leq 0$.

In the sharp $\sigma$-admissible case $\frac{1}{q}+\frac{\sigma}{r}=\frac{\sigma}{2}$ we have

$$
\frac{1}{q^{\prime}}-\frac{1}{q}=-\beta(r, r),
$$

and (14) follows from (17) and the Hardy-Littlewood-Sobolev inequality ([22], Section V.1.2) when $q>q^{\prime}$; that is, when $(q, r) \neq P$.

If we are assuming the truncated decay (3), then (17) can be improved to

$$
\left|\left\langle(U(s))^{*} F(s),(U(t))^{*} G(t)\right\rangle\right| \lesssim(1+|t-s|)^{-1-\beta(r, r)}\|F(s)\|_{r^{\prime}}\|G(t)\|_{r^{\prime}}
$$

and now Young's inequality gives (14) when

$$
-\beta(r, r)+\frac{1}{q}>\frac{1}{q^{\prime}}
$$

or in other words when $(q, r)$ is nonsharp admissible. This concludes the proof of (5), (6) when $(q, r) \neq P$. 
4. The endpoint homogeneous estimate: preliminaries. It remains to prove (5), (6) when

$$
(q, r)=P=\left(2, \frac{2 \sigma}{\sigma-1}\right), \quad \sigma>1
$$

Since $P$ is sharp $\sigma$-admissible, we assume only the untruncated decay (2). This is in fact advantageous because it allows us to use the scaling (12).

It suffices to show (14). By decomposing $T(F, G)$ dyadically as $\sum_{j} T_{j}(F, G)$, where the summation is over the integers $\mathbf{Z}$ and

$$
T_{j}(F, G)=\int_{t-2^{j+1}<s \leq t-2^{j}}\left\langle(U(s))^{*} F(s),(U(t))^{*} G(t)\right\rangle d s d t
$$

we see that it suffices to prove the estimate

$$
\sum_{j}\left|T_{j}(F, G)\right| \lesssim\|F\|_{L_{t}^{2} L_{x}^{r^{\prime}}}\|G\|_{L_{t}^{2} L_{x}^{r^{\prime}}}
$$

In the previous section, (14) was obtained from a one-parameter family of estimates, which came from interpolating between the energy estimate and the decay estimate. This one-parameter family of estimates however is not sufficient to prove the endpoint result, and we will need the following wider two-parameter family of estimates to obtain (22).

Lemma 4.1. The estimate

$$
\left|T_{j}(F, G)\right| \lesssim 2^{-j \beta(a, b)}\|F\|_{L_{t}^{2} L_{x}^{a^{\prime}}}\|G\|_{L_{t}^{2} L_{x}^{b^{\prime}}}
$$

holds for all $j \in \mathbf{Z}$ and all $\left(\frac{1}{a}, \frac{1}{b}\right)$ in a neighbourhood of $\left(\frac{1}{r}, \frac{1}{r}\right)$.

Proof. One can check using (18) and (20) that (23) is invariant under the scaling (12). Thus, it suffices to prove (23) for $j=0$. Since $T_{0}$ is localized in time, we may assume that $F, G$ are supported on a time interval of duration $O(1)$.

We shall prove (23) for the exponents

(i) $a=b=\infty$

(ii) $2 \leq a<r, \quad b=2$

(iii) $\quad 2 \leq b<r, \quad a=2$;

the lemma will then follow by interpolation and the fact that $2<r<\infty$. (See Figure 4.) We remark that when $\sigma=1, r$ becomes infinite and the lemma breaks down at this point. 


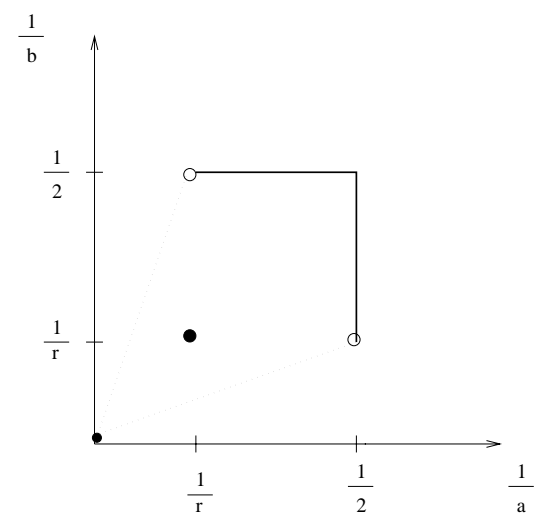

Figure 4. For $\sigma>1,\left(\frac{1}{r}, \frac{1}{r}\right)$ is in the interior of the convex hull of the estimates (i)-(iii).

To prove (i), we integrate (16) in $t$ and $s$ to obtain

$$
\left|T_{0}(F, G)\right| \lesssim\|F\|_{L_{t}^{1} L_{x}^{1}}\|G\|_{L_{t}^{1} L_{x}^{1}}
$$

and (23) follows by Hölder's inequality.

To prove (ii), we bring the $s$-integration inside the inner product in (21) and apply the Cauchy-Schwarz inequality to obtain

$$
\left|T_{j}(F, G)\right| \lesssim\left(\sup _{t}\left\|\int_{t-2^{j+1}<s \leq t-2^{j}}(U(s))^{*} F(s) d s\right\|_{H}\right) \int\left\|(U(t))^{*} G(t)\right\|_{H} d t .
$$

Using the energy estimate $\left\|(U(t))^{*} G(t)\right\|_{H} \lesssim\|G(t)\|_{2}$ this becomes

$$
\left|T_{j}(F, G)\right| \lesssim\left(\sup _{t}\left\|\int_{t-2^{j+1}<s \leq t-2^{j}}(U(s))^{*} F(s) d s\right\|_{H}\right)\|G\|_{L_{t}^{1} L_{x}^{2}}
$$

Define the quantity $q(a)$ by requiring $(q(a), a)$ to be sharp $\sigma$-admissible. By the results of the previous section (6) holds for $(q(a), a)$; applying this to (24) we obtain

$$
\left|T_{0}(F, G)\right| \lesssim\|F\|_{L_{t}^{q(a)^{\prime}} L_{x}^{a^{\prime}}}\|G\|_{L_{t}^{1} L_{x}^{2}}
$$

which by Hölder's inequality gives (23). A similar argument gives (iii).

To finish the proof of the endpoint homogeneous result we have to show that Lemma 4.1 implies (22). We will give a direct proof of this interpolation result in the next section, and an abstract proof using real interpolation theory in Section 6. 
5. Proof of the interpolation step. If one applies Lemma 4.1 directly for $a=r, b=r$ one obtains

$$
\left|T_{j}(F, G)\right| \lesssim\|F\|_{L_{t}^{2} L_{x}^{r^{\prime}}}\|G\|_{L_{t}^{2} L_{x}^{r^{\prime}}}
$$

which clearly won't sum to give (22). However, the fact that we have a twoparameter family of estimates with various exponential decay factors in the neighbourhood of (25) shows that there is room for improvement in (25). To see this in a model case, assume that $F$ and $G$ have the special form

$$
F(t)=2^{-k / r^{\prime}} f(t) \chi_{E(t)}, \quad G(s)=2^{-\tilde{k} / r^{\prime}} g(s) \chi_{\tilde{E}(s)},
$$

where $f, g$ are scalar functions, $k, \tilde{k} \in \mathbf{Z}$ and $E(t), \tilde{E}(s)$ are sets of measure $2^{k}$ and $2^{\tilde{k}}$ respectively for each $t, s$. Then (23) becomes

$$
\left|T_{j}(F, G)\right| \lesssim 2^{-\beta(a, b) j} 2^{-k / r^{\prime}}\|f\|_{2} 2^{k / a^{\prime}} 2^{-\tilde{k} / r^{\prime}}\|g\|_{2} 2^{\tilde{k} / b^{\prime}}
$$

which simplifies using (18) and (20) to

$$
\left|T_{j}(F, G)\right| \lesssim 2^{(k-j \sigma)\left(\frac{1}{r}-\frac{1}{a}\right)+(\tilde{k}-j \sigma)\left(\frac{1}{r}-\frac{1}{b}\right)}\|f\|_{2}\|g\|_{2}
$$

When $a=b=r$ this is just (25). However, since (26) is known to hold for all $\left(\frac{1}{a}, \frac{1}{b}\right)$ in a neighborhood of $\left(\frac{1}{r}, \frac{1}{r}\right)$, we can optimize (26) in $a$ and $b$ and get the improved estimate

$$
\left|T_{j}(F, G)\right| \lesssim 2^{-\varepsilon(|k-j \sigma|+|\tilde{k}-j \sigma|)}\|f\|_{2}\|g\|_{2}
$$

for some $\varepsilon>0$, which does imply (22).

This phenomenon can be viewed as a statement that (25) is only sharp when $F$ and $G$ are both concentrated in a set of size $2^{j \sigma}$. For the wave equation this occurs when $F$ and $G$ resemble the Knapp counterexample

$$
F\left(s,\left(\underline{x}, x_{n}\right)\right)=\psi\left(2^{-j} s, 2^{-j / 2} \underline{x}, x_{n}-s\right) \quad G\left(t,\left(\underline{x}, x_{n}\right)\right)=\psi\left(2^{-j} t-1,2^{-j / 2} \underline{x}, x_{n}-t\right),
$$

(see [25]) and for the Schrödinger equation when $F$ and $G$ have spatial uncertainty $2^{j / 2}$ :

$$
F(s, x)=\psi\left(2^{-j} s, 2^{-j / 2} x\right) \quad G(t, x)=\psi\left(2^{-j} t-1,2^{-j / 2} x\right)
$$

here $\psi$ is a suitable bump function. Thus these examples are in some sense the critical examples for the endpoint Strichartz estimate. However, these examples can only be critical for one scale of $j$, which is why one expects to obtain (22) for general $F, G$ from Lemma 4.1 . 
To apply the above argument in the general case we need to decompose $F$ and $G$ into linear combinations of (approximate) $L^{r^{\prime}}$-normalized characteristic functions. The ability to decompose $F$ and $G$ is an advantage of the bilinear formulation of these estimates. It is difficult to reproduce this argument in the setting of a linear operator estimate.

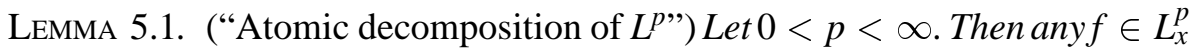
can be written as

$$
f=\sum_{k=-\infty}^{\infty} c_{k} \chi_{k}
$$

where each $\chi_{k}$ is a function bounded by $O\left(2^{-k / p}\right)$ and supported on a set of measure $O\left(2^{k}\right)$, and the $c_{k}$ are non-negative constants such that $\left\|c_{k}\right\|_{p^{p}} \lesssim\|f\|_{p}$.

Proof. Define the distribution function $\lambda(\alpha)$ for $\alpha>0$ by

$$
\lambda(\alpha)=|\{|f(x)|>\alpha\}| .
$$

For each $k$ we set

$$
\begin{aligned}
\alpha_{k} & =\inf _{\lambda(\alpha)<2^{k}} \alpha \\
c_{k} & =2^{k / p} \alpha_{k} \\
\chi_{k} & =\frac{1}{c_{k}} \chi_{\left(\alpha_{k+1}, \alpha_{k}\right]}(|f|) f .
\end{aligned}
$$

The lemma follows easily from the properties of the distribution function (see e.g. [17]). For instance, we prove the bound $\left\|c_{k}\right\|_{p} \lesssim\|f\|_{p}$,

$$
\begin{aligned}
\sum c_{k}^{p} & =\sum 2^{k} \alpha_{k}^{p} \\
& =\int \alpha^{p}\left(\sum 2^{k} \delta_{\alpha_{k}}(\alpha)\right) d \alpha \\
& =\int \alpha^{p}\left(-F^{\prime}(\alpha)\right) d \alpha
\end{aligned}
$$

where

$$
\begin{aligned}
F(\alpha) & =\sum_{k} 2^{k} H\left(\alpha_{k}-\alpha\right) \\
& =\sum_{\alpha_{k}>\alpha} 2^{k} \\
& \leq 2 \lambda(\alpha) .
\end{aligned}
$$


Since $f \in L^{p}$, we may integrate by parts in (28) and use (29) to get

$$
\begin{aligned}
\sum c_{k}^{p} & =p \int \alpha^{p-1} F(\alpha) d \alpha \\
& \lesssim p \int \alpha^{p-1} \lambda(\alpha) d \alpha \\
& =\|f\|_{p}^{p} .
\end{aligned}
$$

By applying Lemma 5.1 with $p=r^{\prime}$ to $F(t)$ and $G(s)$ we have the decomposition

$$
F(t)=\sum_{k} f_{k}(t) \chi_{k}(t), \quad G(s)=\sum_{\tilde{k}} g_{\tilde{k}}(s) \tilde{\chi}_{\tilde{k}}(s),
$$

where for each $t, k$, the function $\chi_{k}(t)$ is bounded by $O\left(2^{-k / r^{\prime}}\right)$ and is supported on a set of measure $O\left(2^{k}\right)$, and similarly for $\tilde{\chi}_{k^{\prime}}(s)$. The functions $f_{k}(t)$ and $g_{\tilde{k}}(s)$ are scalar valued and satisfy the inequalities

$$
\left\|\left(\sum_{k}\left|f_{k}\right|^{r^{\prime}}\right)^{1 / r^{\prime}}\right\|_{L_{t}^{2}} \lesssim\|F\|_{L_{t}^{2} L_{x}^{r^{\prime}}},\left\|\left(\sum_{\tilde{k}}\left|g_{\tilde{k}}\right|^{r^{\prime}}\right)^{1 / r^{\prime}}\right\|_{L_{t}^{2}} \lesssim\|G\|_{L_{t}^{2} L_{x}^{r^{\prime}}}
$$

We are now ready to prove (22). By (29) we have

$$
\sum_{j}\left|T_{j}(F, G)\right| \leq \sum_{j} \sum_{k} \sum_{\tilde{k}}\left|T_{j}\left(f_{k} \chi_{k}, g_{\tilde{k}} \tilde{\chi}_{\tilde{k}}\right)\right|
$$

But by the analysis at the start of this section we have

$$
\left|T_{j}\left(f_{k} \chi_{k}, g_{\tilde{k}} \tilde{\chi}_{\tilde{k}}\right)\right| \lesssim 2^{-\varepsilon(|k-j \sigma|+|\tilde{k}-j \sigma|)}\left\|f_{k}\right\|_{2}\left\|g_{\tilde{k}}\right\|_{2}
$$

Combining these two inequalities and summing in $j$ we obtain

$$
\sum_{j}\left|T_{j}(F, G)\right| \lesssim \sum_{k} \sum_{\tilde{k}}(1+|k-\tilde{k}|) 2^{-\varepsilon|k-\tilde{k}|}\left\|f_{k}\right\|_{2}\left\|g_{\tilde{k}}\right\|_{2}
$$

Since the quantity $(1+|k|) 2^{-\varepsilon|k|}$ is absolutely summable, we may apply Young's inequality and obtain

$$
\sum_{j}\left|T_{j}(F, G)\right| \lesssim\left(\sum_{k}\left\|f_{k}\right\|_{2}^{2}\right)^{1 / 2}\left(\sum_{\tilde{k}}\left\|g_{\tilde{k}}\right\|_{2}^{2}\right)^{1 / 2}
$$


Interchanging the $L^{2}$ and $l^{2}$ norms and using the inclusion $l^{\prime} \subset l^{2}$ we obtain

$$
\sum_{j}\left|T_{j}(F, G)\right| \lesssim\left\|\left(\sum_{k}\left|f_{k}\right|^{r^{\prime}}\right)^{1 / r^{\prime}}\right\|_{2}\left\|\left(\sum_{\tilde{k}}\left|g_{\tilde{k}}\right|^{r^{\prime}}\right)^{1 / r^{\prime}}\right\|_{2},
$$

and (22) follows by (30).

The use of the inclusion $l^{r^{\prime}} \subset l^{2}$ shows that there is a slight amount of "slack" in this argument. In fact, the $L_{x}^{r}$ norm in (5) can be sharpened to a Lorentz space norm $L_{x}^{r, 2}$. (See the argument in the next section, and the remarks at the end of the paper.)

6. Alternate proof of the interpolation step. In this section we rephrase the above derivation of (22) from Lemma 4.1 using existing results in real interpolation theory. Our notation follows [1] and [26].

Let $A_{0}, A_{1}$ be Banach spaces. (We will always assume that any pair of Banach spaces $A_{0}, A_{1}$ can be contained in some larger Banach space $A$.) We define the real interpolation spaces $\left(A_{0}, A_{1}\right)_{\theta, q}$ for $0<\theta<1,1 \leq q \leq \infty$ via the norm

$$
\|a\|_{\left(A_{0}, A_{1}\right)_{\theta, q}}=\left(\int_{0}^{\infty}\left(t^{-\theta} K(t, a)\right)^{q} \frac{d t}{t}\right)^{1 / q},
$$

where

$$
K(t, a)=\inf _{a=a_{0}+a_{1}}\left\|a_{0}\right\|_{A_{0}}+t\left\|a_{1}\right\|_{A_{1}} .
$$

We will need the interpolation space identities

$$
\left(L_{t}^{2} L_{x}^{p_{0}}, L_{t}^{2} L_{x}^{p_{1}}\right)_{\theta, 2}=L_{t}^{2} L_{x}^{p, 2}
$$

whenever $p_{0} \neq p_{1}$ and $\frac{1}{p}=\frac{1-\theta}{p_{0}}+\frac{\theta}{p_{1}}$, (see [26] Sections 1.18.2 and 1.18.6), and

$$
\left(l_{\infty}^{s_{0}}, l_{\infty}^{s_{1}}\right)_{\theta, 1}=l_{1}^{s}
$$

whenever $s_{0} \neq s_{1}$ and $s=(1-\theta) s_{0}+\theta s_{1}$ (see [1] Section 5.6), where $l_{q}^{s}=$ $L^{q}\left(\mathbf{Z}, 2^{j s} d j\right)$ are weighted sequence spaces and $d j$ is counting measure.

The bilinear interpolation we will use is the following:

Lemma 6.1. ([1], Section 3.13.5(b)) If $A_{0}, A_{1}, B_{0}, B_{1}, C_{0}, C_{1}$ are Banach spaces, and the bilinear operator $T$ is bounded from

$$
\begin{aligned}
& T: A_{0} \times B_{0} \rightarrow C_{0} \\
& T: A_{0} \times B_{1} \rightarrow C_{1} \\
& T: A_{1} \times B_{0} \rightarrow C_{1},
\end{aligned}
$$


then whenever $0<\theta_{0}, \theta_{1}<\theta<1,1 \leq p, q, r \leq \infty$ are such that $1 \leq \frac{1}{p}+\frac{1}{q}$ and $\theta=\theta_{0}+\theta_{1}$, one has

$$
T:\left(A_{0}, A_{1}\right)_{\theta_{0}, p r} \times\left(B_{0}, B_{1}\right)_{\theta_{1}, q r} \rightarrow\left(C_{0}, C_{1}\right)_{\theta, r} .
$$

The estimate (23) can be rewritten as

$$
T: L_{t}^{2} L_{x}^{a^{\prime}} \times L_{t}^{2} L_{x}^{b^{\prime}} \rightarrow l_{\infty}^{\beta(a, b)}
$$

where $T=\left\{T_{j}\right\}$ is the vector-valued bilinear operator corresponding to the $T_{j}$.

We apply Lemma 6.1 to (31) with $r=1, p=q=2$ and arbitrary exponents $a_{0}, a_{1}, b_{0}, b_{1}$ such that

$$
\beta\left(a_{0}, b_{1}\right)=\beta\left(a_{1}, b_{0}\right) \neq \beta\left(a_{0}, b_{0}\right) .
$$

Using the real interpolation space identities mentioned above we obtain

$$
T: L_{t}^{2} L_{x}^{a^{\prime}, 2} \times L_{t}^{2} L_{x}^{b^{\prime}, 2} \rightarrow l_{1}^{\beta(a, b)}
$$

for all $(a, b)$ in a neighbourhood of $(r, r)$. Applying this to $a=b=r$ and using the fact that $L^{r^{\prime}} \subset L^{r^{\prime}, 2}$ we obtain

$$
T: L_{t}^{2} L_{x}^{r^{\prime}} \times L_{t}^{2} L_{x}^{r^{\prime}} \rightarrow l_{1}^{0}
$$

which is (22), as desired.

7. The retarded estimate. Having completed the proof of the homogeneous estimate (5), we turn to the retarded estimate (7). By duality and (15) the estimate is equivalent to

$$
|T(F, G)| \lesssim\|F\|_{L_{t}^{q^{\prime}} L_{x}^{r^{\prime}}}\|G\|_{L_{t}^{\tilde{q}^{\prime}} L_{x}^{\tilde{r}^{\prime}}}
$$

By repeating the argument used to prove (24), we have

$$
|T(F, G)| \lesssim\left(\sup _{t}\left\|\int_{s<t}(U(s))^{*} F(s) d s\right\|_{H}\right)\|G\|_{L_{t}^{1} L_{x}^{2}}
$$

and when $(\tilde{q}, \tilde{r})=(\infty, 2)$ the estimate (32) follows from (6). Similarly one has (32) when $(q, r)=(\infty, 2)$. From (14) we see that (32) holds when $(q, r)=(\tilde{q}, \tilde{r})$.

By interpolating between these three special cases one can obtain the result whenever $\left(\frac{1}{q}, \frac{1}{r}\right),\left(\frac{1}{\tilde{q}}, \frac{1}{\tilde{r}}\right)$, and $\left(\frac{1}{\infty}, \frac{1}{2}\right)$ are collinear. In particular, we get (32) whenever $(q, r)$ and $(\tilde{q}, \tilde{r})$ are both sharp $\sigma$-admissible. This concludes the proof of (7) under the untruncated decay hypothesis. 
It remains to consider the case when we have the truncated decay hypothesis (3) and at least one of $(q, r),(\tilde{q}, \tilde{r})$ is nonsharp $\sigma$-admissible. (In the concrete context of the wave equation one can obtain this case from the previous ones by simply using Sobolev imbedding.) Since every $\sigma$-admissible pair $(q, r)$ is an interpolant between $(q, \infty)$ and a sharp $\sigma$-admissible pair it suffices to consider the case when $r=\infty$ or $\tilde{r}=\infty$. Without loss of generality we may assume that $r=\infty$.

We first dispose of the case $\tilde{q}=\infty$. From (19) for $s=t$ and $F(s)=G(t)$, we obtain the estimate $\left\|(U(t))^{*} G(t)\right\|_{H} \lesssim\|G(t)\|_{\tilde{r}^{\prime}}$. Inserting this as a substitute for the energy estimate in the derivation of (33), we obtain

$$
|T(F, G)| \lesssim\left(\sup _{t}\left\|\int_{s<t}(U(s))^{*} F(s) d s\right\|_{H}\right)\|G\|_{L_{t}^{1} L_{x}^{\tilde{r}^{\prime}}}
$$

and (32) follows from (6). Thus we may assume that $\tilde{q}<\infty$. Note that this implies $\tilde{r}>2$, by (4).

To deal with the remaining cases it suffices to prove the following crude variant of Lemma 4.1; the estimate (32) will follow by optimizing (34) below in $\alpha$ and using the triangle inequality.

LEMMA 7.1. The estimate

$$
\left|T_{j}(F, G)\right| \lesssim 2^{\alpha j}\|F\|_{L_{t}^{q^{\prime}} L_{x}^{1}}\|G\|_{L_{t}^{\tilde{q}^{\prime}} L_{x}^{a^{\prime}}}
$$

holds for $a=\tilde{r}$ and all $\alpha$ in a neighbourhood of 0 .

Proof. By localization we may assume that $F$ and $G$ are supported in a time interval of duration $O\left(2^{j}\right)$. We first prove (34) for the pairs:

(i) $\quad a=2, \alpha=\frac{1}{\tilde{q}}$

(ii) $\quad a=\infty, \alpha=-\sigma+\frac{1}{q}+\frac{1}{\tilde{q}}$

(iii) $a=\infty, \alpha=\frac{1}{q}+\frac{1}{\tilde{q}}$.

To prove (i), we apply (6) for $(q, \infty)$ to $(24)$ to obtain

$$
\left|T_{j}(F, G)\right| \lesssim\|F\|_{L_{t}^{q^{\prime}} L_{x}^{1}}\|G\|_{L_{t}^{1} L_{x}^{2}}
$$

and (34) for (i) follows by Hölder's inequality.

Next, we integrate (16) in $s$ and $t$ to obtain

$$
\left|T_{j}(F, G)\right| \lesssim 2^{-j \sigma}\|F\|_{L_{t}^{1} L_{x}^{1}}\|G\|_{L_{t}^{1} L_{x}^{1}}
$$

and (34) for (ii) follows by Hölder's inequality. To handle (iii) we note that (3) implies (16) with $\sigma$ replaced by 0 , and so we can repeat the argument in (ii). By interpolating between (i) and (iii) we obtain (34) for $a=\tilde{r}$ and some positive $\alpha$. 
By interpolating between (i) and (ii) we obtain (34) for $a=\tilde{r}$ and

$$
\begin{aligned}
\alpha & =\left(1-\frac{2}{\tilde{r}}\right)\left(-\sigma+\frac{1}{q}\right)+\frac{1}{\tilde{q}} \\
& =-\left(1-\frac{2}{\tilde{r}}\right)\left(\frac{\sigma}{2}-\frac{\sigma}{\infty}-\frac{1}{q}\right)-\left(\frac{\sigma}{2}-\frac{\sigma}{\tilde{r}}-\frac{1}{\tilde{q}}\right)
\end{aligned}
$$

which is negative by (4), the assumption $\tilde{r}>2$, and the fact that at least one of $(q, \infty),(\tilde{q}, \tilde{r})$ is nonsharp admissible. By interpolating between both values of $\alpha$ we obtain our result.

Note that the admissibility of $(\tilde{q}, \tilde{r})$ was only used in the above lemma to ensure that the quantity (36) was negative. However, (36) can be negative even for inadmissible $(\tilde{q}, \tilde{r})$. Thus, for example, the estimate (7) holds for $\sigma=1$, the admissible indices $q=4, r=\infty$, and the inadmissible indices $\tilde{q}=4,3<\tilde{r}<4$. Hence the inhomogeneous estimates have a wider range of admissibility than the homogeneous estimates.

It seems of interest to determine all pairs of exponents $(q, r),(\tilde{q}, \tilde{r})$ for which (7) holds; the range of exponents given by the above arguments are certainly not optimal. The problem is likely to be very difficult; in the case of the wave equation the estimate (7) for general pairs of exponents is related to unsolved conjectures such as the local smoothing conjecture of [19] and the Bochner-Riesz problem for cone multipliers (see [2]).

8. Strichartz estimates for the wave and Schrödinger equations. We start with showing the necessity of the various conditions in Corollary 1.3. The gap condition follows from dimensional analysis (scaling considerations). The admissibility conditions

$$
\frac{1}{q}+\frac{(n-1) / 2}{r} \leq \frac{(n-1) / 2}{2}, \quad \frac{1}{\tilde{q}}+\frac{(n-1) / 2}{\tilde{r}} \leq \frac{(n-1) / 2}{2}
$$

follow from the Knapp counterexample for the cone and its adjoint, whereas the inadmissibility of $(q, r, n)=(2, \infty, 3)$ or $(\tilde{q}, \tilde{r}, n)=(2, \infty, 3)$ was shown in [12]. The remaining admissibility conditions

$$
q \geq q^{\prime}, \quad \tilde{q} \geq \tilde{q}^{\prime}
$$

follow from the following translation invariance argument. The (homogeneous part of the) estimate can be viewed as an operator boundedness result from $\dot{H}^{\alpha}$ to $L_{t}^{q} L_{x}^{r}$, and by the $T T^{*}$ method this is equivalent to an operator boundedness result from $L_{t}^{q^{\prime}} L_{x}^{r^{\prime}}$ to $L_{t}^{q} L_{x}^{r}$. However, in the limiting case $T=\infty$, this operator is time-translation invariant, and so cannot map a higher-exponent space to a 
lower-exponent space (see [8]). Thus $q \geq q^{\prime}$, and a dual version of the same argument gives $\tilde{q} \geq \tilde{q}^{\prime}$.

Now suppose that $\alpha, q, r$ satisfy the conditions of the theorem, and that $u$ is a solution to (8). We use Duhamel's principle to write $u$ as

$$
u(t)=S(t)(f, g)+\mathcal{G} F(t)
$$

where

$$
\begin{aligned}
S(t)(f, g) & =\cos (t \sqrt{-\Delta}) f+\frac{\sin (t \sqrt{-\Delta})}{\sqrt{-\Delta}} g \\
\mathcal{G} F(t) & =\int_{0}^{t} \frac{\sin ((t-s) \sqrt{-\Delta})}{\sqrt{-\Delta}} F(s) d s
\end{aligned}
$$

are the homogenous and inhomogenous components of $u$.

By the usual reduction using Littlewood-Paley theory we may assume that the spatial Fourier transform of $f, g, F$ (and consequently $u$ ) are all localized in the annulus $\left\{|\xi| \sim 2^{j}\right\}$ for some $j$. (See Lemma 5.1 of [21] and the subsequent discussion. The cases $r=\infty$ or $\tilde{r}=\infty$ can also be treated by this argument, but the Lebesgue spaces $L_{x}^{r}, L_{x}^{\tilde{r}^{\prime}}$ must be replaced by their Besov space counterparts.) By the gap condition, the estimate is scale invariant, and so we may assume $j=0$. Now that frequency is localized, $\sqrt{-\Delta}$ becomes an invertible smoothing operator, and we may replace the Sobolev norms $\dot{H}^{\gamma}, \dot{H}^{\gamma-1}$ with the $L^{2}$ norm. Combining these reductions with (36) and (37), we see that (9) will follow from the estimates

$$
\begin{aligned}
\left\|U_{ \pm}(t) f\right\|_{C\left(L_{x}^{2}\right)} & \lesssim\|f\|_{2} \\
\left\|U_{ \pm}(t) f\right\|_{L_{t}^{q} L_{x}^{r}} & \lesssim\|f\|_{2} \\
\left\|U_{ \pm}(t) g\right\|_{C\left(L_{x}^{2}\right)} & \lesssim\|g\|_{2} \\
\left\|U_{ \pm}(t) g\right\|_{L_{t}^{q} L_{x}^{r}} & \lesssim\|g\|_{2} \\
\left\|\int_{t>s} U_{ \pm}(t)\left(U_{ \pm}(s)\right)^{*} F(s) d s\right\|_{C\left(L^{2}\right)} & \lesssim\|F\|_{L^{q^{\prime}}{ }_{t} L_{x}^{\tilde{r}^{\prime}}} \\
\left\|\int_{t>s} U_{ \pm}(t)\left(U_{ \pm}(s)\right)^{*} F(s) d s\right\|_{L_{t}^{q} L_{x}^{r}} & \lesssim\|F\|_{L_{t}^{\tilde{q}^{\prime}} L_{x}^{\tilde{r}^{\prime}}}
\end{aligned}
$$

where the truncated wave evolution operators $U_{ \pm}(t)$ are given by

$$
\widehat{U_{ \pm}(t) f}(\xi)=\chi_{[0, T]}(t) \beta(\xi) e^{ \pm i t|\xi|} \hat{f}(\xi)
$$

for some Littlewood-Paley cutoff function $\beta$ supported on $|\xi| \sim 1$. 
Let us temporarily replace the $C\left(L_{x}^{2}\right)$ norm in the above by the $L_{t}^{\infty} L_{x}^{2}$ norm. All of the above estimates will follow from Theorem 1.2 with $H=L^{2}\left(\mathbf{R}^{n}\right), X=\mathbf{R}^{n}$, $\sigma=(n-1) / 2$, once we show that $U_{ \pm}$obeys the energy estimate (1) and the truncated decay estimate (3). The former estimate is immediate from Plancherel's theorem, and the latter follows from standard stationary phase estimates on the kernel of $U_{ \pm}(t)\left(U_{ \pm}(s)\right)^{*}$. (See [20], pp. 223-224.)

We now address the question of continuity in $L^{2}$. The continuity of $U_{ \pm}(t) f$ and $U_{ \pm}(t) g$ follow from Plancherel's theorem. To show that the quantity

$$
\mathcal{G}_{ \pm} F(t)=\int_{t>s} U_{ \pm}(t)\left(U_{ \pm}(s)\right)^{*} F(s) d s
$$

is continuous in $L^{2}$, one can use the identity

$$
\mathcal{G}_{ \pm} F(t+\varepsilon)=e^{i \varepsilon \sqrt{-\Delta}} \mathcal{G}_{ \pm} F(t)+\mathcal{G}_{ \pm}\left(\chi_{[t, t+\varepsilon]} F\right)(t),
$$

the continuity of $e^{i \varepsilon \sqrt{-\Delta}}$ as an operator on $L^{2}$, and the fact that

$$
\left\|\chi_{[t, t+\varepsilon]} F\right\|_{L_{t}^{\tilde{q}^{\prime}} L_{x}^{\tilde{r}^{\prime}}} \rightarrow 0 \text { as } \varepsilon \rightarrow 0 .
$$

The proof of Corollary 1.4 proceeds similarly, but without the additional technicalities involving Littlewood-Paley theory. From the scaling $x \leftarrow \lambda x, t \leftarrow$ $\lambda^{2} t$ and the same translation invariance argument as before, together with the negative result in [16] for $q=2, r=\infty, n=2$ we see that the conditions on $q, r$ are necessary. For sufficiency, we write $u$ as $u=S f-i \mathcal{G F}$, where

$$
\begin{aligned}
S(t)(f) & =\chi_{[0, T]}(t) e^{i t \Delta} f \\
\mathcal{G} F(t) & =\int_{t>s} S(t)(S(s))^{*} F(s) d s,
\end{aligned}
$$

and apply Theorem 1.2 with $H=L^{2}\left(\mathbf{R}^{n}\right), X=\mathbf{R}^{n}, \sigma=n / 2$. The energy estimate

$$
\left\|e^{i t \Delta} f\right\|_{2} \lesssim\|f\|_{2}
$$

follows from Plancherel's theorem as before, and the untruncated decay estimate

$$
\left\|e^{i(t-s) \Delta} f\right\|_{\infty} \lesssim|t-s|^{-n / 2}\|f\|_{1}
$$

follows from the explicit representation of the Schrödinger evolution operator

$$
e^{i t \Delta} f(x)=\frac{1}{(2 \pi i t)^{n / 2}} \int_{\mathbf{R}^{n}} e^{-\frac{|x-y|^{2}}{2 i t}} f(y) d y .
$$

The proof of continuity in $L^{2}$ proceeds in analogy with the wave equation. 


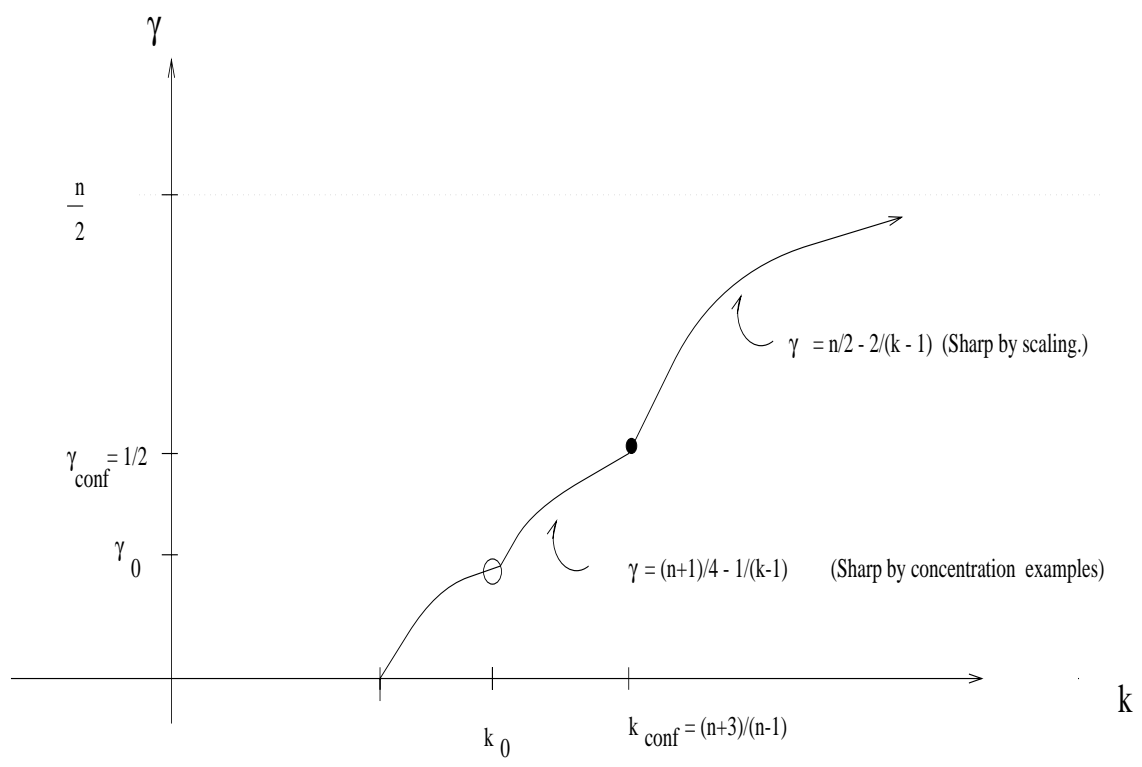

Figure 5. A sketch of known well-posedness results in $n \geq 4$.

9. Application to a semi-linear wave equation. Following the notation of Lindblad-Sogge [14], we consider the initial value problem

$$
\begin{aligned}
\left(-\frac{\partial^{2}}{\partial t^{2}}+\Delta\right) u & =F_{k}(u) \\
u(x, 0) & =f(x) \in \dot{H}^{\gamma}\left(\mathbf{R}^{n}\right) \\
\partial_{t} u(x, 0) & =g(x) \in \dot{H}^{\gamma-1}\left(\mathbf{R}^{n}\right)
\end{aligned}
$$

where $u$ is scalar or vector valued, $k>1$ and the nonlinearity $F_{k} \in C^{1}$ satisfies

$$
\begin{aligned}
\left|F_{k}(u)\right| & \lesssim|u|^{k} \\
|u|\left|F_{k}^{\prime}(u)\right| & \sim\left|F_{k}(u)\right| .
\end{aligned}
$$

The question of how much regularity $\gamma=\gamma(k, n)$ is needed to insure local well-posedness of (38) was addressed for higher dimensions and nonlinearities in [10]; and then almost completely answered in [14]. (See [13] for $n=3, k=2$.) The purpose of this section is to simply show the new endpoint estimate in Corollary 1.3 above gives a new "endpoint" well-posedness result for (38) in dimensions $n \geq 4$.

The results of [14] in dimensions $n \geq 4$ are sketched in Figure 5. (Those positive results dealing with $k_{0}<k<\frac{n+2}{n-2}$ were obtained in [10] as well, using a different argument.) 
The piecewise smooth curve in the figure represents the smallest known $\gamma$ for which (38) is locally well-posed. When

$$
k>k_{0}=\frac{(n+1)^{2}}{(n-1)^{2}+4}
$$

it is shown in [14] that the results are best-possible. For $k<k_{0}$, the sharpness is not known, but [14] proves local well-posedness for

$$
\gamma=\frac{n+1}{4}-\frac{(n+1)(n+5)}{4} \cdot \frac{1}{2 n k-(n+1)} .
$$

In this section, we simply extend the well-posedness results to include the case $k=k_{0}$.

COROllary 9.1. Assume $n \geq 4$ and

$$
\begin{aligned}
& \gamma=\gamma_{0}=\frac{n-3}{2(n-1)} \\
& k=k_{0}=\frac{(n+1)^{2}}{(n-1)^{2}+4} .
\end{aligned}
$$

Then there is a $T>0$ depending only on $\|f\|_{\dot{H}^{\gamma}}+\|g\|_{\dot{H}^{\gamma-1}}$ and a unique weak solution u to (38) with

$$
u \in L_{t}^{q_{0}} L_{x}^{r_{0}}\left([0, T] \times \mathbf{R}^{n}\right)
$$

where

$$
q_{0}=\frac{2(n+1)}{n-3} \quad r_{0}=\frac{2\left(n^{2}-1\right)}{(n-1)^{2}+4} .
$$

In addition, the solution satisfies

$$
u \in C\left([0, T], \dot{H}^{\gamma}\right) \cap C^{1}\left([0, T], \dot{H}^{\gamma-1}\right)
$$

and depends continuously (in the norms (40)-(41)) on the data.

Proof. The argument will rely on the estimate (9) applied to the exponents

$$
\gamma=\gamma_{0}, \quad(q, r)=\left(q_{0}, r_{0}\right), \quad(\tilde{q}, \tilde{r})=P=\left(2, \frac{2(n-1)}{n-3}\right)
$$

one may easily check that $(q, r),(\tilde{q}, \tilde{r})$ are wave-admissible and obey the gap condition. The only other properties of these exponents we shall use are that $r=\tilde{r}^{\prime} k$ and $q>\tilde{q}^{\prime} k$. (For the endpoint $k=k_{0}, \gamma=\gamma_{0}$ these requirements uniquely determine $(q, r)$ and $(\tilde{q}, \tilde{r})$.) 
We apply the standard fixed point argument (see in particular the presentation in [4]) in the space

$$
X=X(T, M)=\left\{u \in L_{t}^{q}\left([0, T] ; L_{x}^{r}\right) \mid\|u\|_{L_{t}^{q}\left([0, T] ; L_{x}^{r}\right)} \leq M\right\}
$$

with $T$ and $M$ to be determined. By (36), the problem of finding a solution $u$ of (38) is equivalent to finding a fixed point of the mapping

$$
\mathcal{F} u(t)=S(t)(f, g)+\mathcal{G} F_{k_{0}}(u)
$$

Accordingly, we will find $M, T$ so that $\mathcal{F}$ is a contraction on $X(T, M)$. It will suffice to show that for all $M$ there is a $T>0$ so that

$$
\|\mathcal{F} u-\mathcal{F} \mathrm{v}\|_{X} \leq \frac{1}{2}\|u-\mathrm{v}\|_{X}
$$

The fact that $\mathcal{F}: X \longrightarrow X$ follows by picking $M$ large enough so

$$
\|\mathcal{F} 0\|_{X} \leq \frac{M}{2}
$$

note that $\|\mathcal{F} 0\|_{X}$ is finite by (9) applied to the homogeneous problem.

By (9) we have

$$
\begin{aligned}
\|\mathcal{F} u-\mathcal{F} \mathrm{V}\|_{X} & =\left\|\mathcal{G}\left(F_{k}(u)-F_{k}(\mathrm{v})\right)\right\|_{X} \\
& \lesssim\left\|F_{k}(u)-F_{k}(\mathrm{v})\right\|_{L_{t}^{\tilde{q}^{\prime}} L_{x}^{\tilde{r}^{\prime}}}
\end{aligned}
$$

The assumptions (39) give

$$
\begin{aligned}
\left|F_{k}(u)-F_{k}(\mathrm{v})\right| & =\left|\int_{0}^{1} \frac{d}{d \lambda} F_{k}(\lambda u+(1-\lambda) \mathrm{v}) d \lambda\right| \\
& =\left|\int_{0}^{1}(u-\mathrm{v}) \cdot \nabla F_{k}(\lambda u+(1-\lambda) \mathrm{v}) d \lambda\right| \\
& \lesssim|u-\mathrm{v}|(|u|+|\mathrm{v}|)^{k-1}
\end{aligned}
$$

Using this in (47) gives

$$
\|\mathcal{F} u-\mathcal{F} \mathrm{V}\|_{X} \lesssim\left\||u-\mathrm{v}|(|u|+|\mathrm{v}|)^{k-1}\right\|_{L_{t}^{\tilde{q}^{\prime}} L_{x}^{\tilde{r}^{\prime}}}
$$


However, by the generalized Hölder inequality we have

$$
\begin{aligned}
\||u-\mathrm{v}| & (|u|+|\mathrm{v}|)^{k-1} \|_{L_{t}^{\tilde{q}^{\prime}} L_{x}^{\tilde{r}^{\prime}}} \\
& \leq\|u-\mathrm{v}\|_{L_{t}^{q} L_{x}^{r}}\left\|(|u|+|\mathrm{v}|)^{k-1}\right\|_{L_{t}^{q /(k-1)} L_{x}^{r /(k-1)}}\left\|\chi_{[0, T]}\right\|_{L_{t}^{p} L_{x}^{\infty}},
\end{aligned}
$$

where $1 \leq p<\infty$ is chosen so that

$$
\frac{1}{\tilde{q}^{\prime}}=\frac{1}{q}+\frac{1}{q /(k-1)}+\frac{1}{p}, \quad \frac{1}{\tilde{r}^{\prime}}=\frac{1}{r}+\frac{1}{r /(k-1)}+\frac{1}{\infty}
$$

note that $p$ is well-defined since $r=\tilde{r}^{\prime} k, q>\tilde{q}^{\prime} k$. (In the endpoint case $k=k_{0}$, $\gamma=\gamma_{0}$ we have $p=\frac{n^{2}-2 n+5}{8}$.)

By the assumptions on $u, \mathrm{v}$ the estimate (48) simplifies to

$$
\left\||u-\mathrm{v}|(|u|+|\mathrm{v}|)^{k-1}\right\|_{L_{t}^{\tilde{q}^{\prime}} L_{x}^{\tilde{r}^{\prime}}} \lesssim T^{1 / p} M^{k-1}\|u-\mathrm{v}\|_{X}
$$

Thus if we choose $T$ so that $T^{1 / p} M^{k-1} \ll 1$, then (47) and (49) give the desired contraction (44).

To obtain the regularity (41) for $u$ we apply (49) with $\mathrm{V}=0$ to obtain

$$
\left\|F_{k}(u)\right\|_{L_{t}^{\tilde{q}^{\prime}} L_{x}^{\tilde{r}^{\prime}}} \leq T^{1 / p} M^{k-1}\|u\|_{X}<\infty
$$

and (41) follows from (9).

Finally, we need to show uniqueness. (Continuous dependence on the data is similarly included in the above arguments.) Suppose that we have two solutions $u, \mathrm{v}$ to $(38)$ for time $\left[0, T^{*}\right]$ such that

$$
\|u\|_{L_{t}^{q}\left(\left[0, T^{*}\right] ; L_{x}^{r}\right)},\|\mathrm{V}\|_{L_{t}^{q}\left(\left[0, T^{*}\right] ; L_{x}^{r}\right)} \leq M
$$

for some $M$. Choose $0<T \leq T^{*}$ such that $T^{1 / p} M^{k-1} \ll 1$. By the above arguments (44) holds, which implies that $u=\mathrm{v}$ for time [0,T]. Since $T$ depends only on $M$, we may iterate this argument and obtain $u=\mathrm{V}$ for all times $\left[0, T^{*}\right]$.

10. Further remarks. An inspection of the proof of Theorem 1.2, especially the abstract interpolation step in Section 6, shows that the Lebesgue spaces $L_{X}^{r}$ in the hypotheses of Theorem 1.2 can be replaced by an interpolation family of abstract Banach spaces. More precisely, we have: 
THEOREM 10.1. Let $\sigma>0, H$ be a Hilbert space and $B_{0}, B_{1}$ be Banach spaces. Suppose that for each time $t$ we have an operator $U(t): H \rightarrow B_{0}^{*}$ such that

$$
\begin{aligned}
\|U(t)\|_{H \rightarrow B_{0}^{*}} & \lesssim 1 \\
\left\|U(t)(U(s))^{*}\right\|_{B_{1} \rightarrow B_{1}^{*}} & \lesssim|t-s|^{-\sigma} .
\end{aligned}
$$

Let $B_{\theta}$ denote the real interpolation space $\left(B_{0}, B_{1}\right)_{\theta, 2}$. Then we have the estimates

$$
\begin{aligned}
\|U(t) f\|_{L_{t}^{q} B_{\theta}^{*}} & \lesssim\|f\|_{H} \\
\left\|\int(U(s))^{*} F(s) d s\right\|_{H} & \lesssim\|F\|_{L_{t}^{q^{\prime}} B_{\theta}} \\
\left\|\int_{s<t} U(t)(U(s))^{*} F(s) d s\right\|_{L_{t}^{q} B_{\theta}^{*}} & \lesssim\|F\|_{L_{t}^{\tilde{q}^{\prime}} B_{\tilde{\theta}}^{*}}
\end{aligned}
$$

whenever $0 \leq \theta \leq 1,2 \leq q=\frac{2}{\sigma \theta},(q, \theta, \sigma) \neq(2,1,1)$, and similarly for $(\tilde{q}, \tilde{\theta})$. If the decay estimate is strengthened to

$$
\left\|U(t)(U(s))^{*}\right\|_{B_{1} \rightarrow B_{1}^{*}} \lesssim(1+|t-s|)^{-\sigma}
$$

then the requirement $q=\frac{2}{\sigma \theta}$ can be relaxed to $q \geq \frac{2}{\sigma \theta}$, and similarly for $(\tilde{q}, \tilde{\theta})$.

Thus, for instance, one can formulate a version of Theorem 1.2 for Besov spaces instead of Lebesgue spaces; this allows a slightly shorter proof of Corollary 1.3, using the interpolation theory of Besov spaces to avoid an explicit mention of Littlewood-Paley theory (cf. the approach in [7]).

Theorems 1.2 and 10.1 can be applied to higher-dimensional problems other than the wave and Schrödinger equations. (These theorems are also valid in the low-dimensional case $\sigma \leq 1$, but their content is not new for this case.) For example, in [11] there is the following Strichartz (or "global smoothing") result (in our notation):

TheOREM 10.2. ([11], Theorem 3.1) Let $P$ be a real elliptic polynomial of degree $m$ in $\mathbf{R}^{n}$. Then

$$
\begin{aligned}
\left\|W_{\gamma}(t) f\right\|_{L_{t}^{q} L_{x}^{r}} & \lesssim\|f\|_{2} \\
\left\|\int_{s<t} W_{\gamma}(t) W_{\gamma}^{*}(s) F(s) d s\right\|_{L_{t}^{q} L_{x}^{r}} & \lesssim\|F\|_{L_{t}^{q^{\prime}} L_{x}^{r^{\prime}}}
\end{aligned}
$$


where $\gamma=2 / n q,(q, r)$ is sharp $n / 2$-admissible, $q<2$,

$$
W_{\gamma}(t) f(x)=\int e^{i(t P(\xi)+x \cdot \xi)}\left|\operatorname{det}\left(P_{\xi \xi}\right)\right|^{\gamma / 2} \hat{f}(\xi) \psi_{P}(\xi) d \xi
$$

and $\psi_{P}$ is a suitable cutoff function.

By Theorem 1.2 we can remove the restriction $q<2$ (provided that $n>2$ ) in the above theorem, and generalize the retarded estimate to two different admissible pairs $(q, r),(\tilde{q}, \tilde{r})$ of exponents. The proof proceeds along analogous lines to that of Corollary 1.3 (using either Littlewood-Paley theory or Theorem 10.1 for Sobolev spaces to handle the $\gamma$ parameter). The energy estimate follows from Plancherel's theorem, and the decay estimate is proven in Lemma 3.5 of [11]. We omit the details.

As observed in [11], it seems likely that the above results can be partially extended to the case when the symbol $P(\xi)$ is not elliptic, or even polynomial.

We now consider Strichartz estimates for the kinetic transport equation

$$
\left\{\begin{array}{l}
\frac{\partial}{\partial t} f(t, x, \xi)+\xi \cdot \nabla_{x} f(t, x, \xi)=0 \quad(t, x, \xi) \in \mathbf{R} \times \mathbf{R}^{n} \times \mathbf{R}^{n} \\
f(0, x, \xi)=f^{0}(x, \xi)
\end{array}\right.
$$

Given (nonnegative) $f$ and $f_{0}$ as above, we seek all estimates of the form

$$
\|f\|_{L_{t}^{q} L_{x}^{p} L_{\xi}^{r}} \lesssim\left\|f^{0}\right\|_{L_{x, \xi}^{a}}
$$

By dimensional analysis the following conditions are necessary:

$$
\frac{2}{q}=n\left(\frac{1}{r}-\frac{1}{p}\right), \quad \frac{1}{a}=\frac{1}{2}\left(\frac{1}{r}+\frac{1}{p}\right) .
$$

By spatial translation invariance we must have $p \geq a$. By considering the counterexample $f_{0}=\sum_{j=1}^{N} \chi_{E_{j}}$, where

$$
E_{j}=\left\{(x, \xi):|x| \sim 2^{j},\left|\xi+2^{-j} x\right| \sim 2^{-j}\right\}
$$

and noting that the solution $f$ satisfies

$$
f(t, x, \xi)=1 \text { whenever }|x|,|\xi| \ll 1,\left|t-2^{j}\right| \ll 1,1 \leq j \leq N \text {, }
$$

we see that we must have $q \geq a$.

THEOREM 10.3. ([3], Theorem 1(b)) If the above necessary conditions hold and $q>2 \geq a$, then (50) holds. 
Since the problem is invariant under the transformation

$$
f_{0} \leftarrow f_{0}^{\alpha}, \quad f \leftarrow f^{\alpha}, \quad(q, p, r, a) \leftarrow\left(\frac{q}{\alpha}, \frac{p}{\alpha}, \frac{r}{\alpha}, \frac{a}{\alpha}\right)
$$

one can easily replace the restriction $q>2 \geq a$ in the above theorem with $q>a$. (Thus, for instance, Theorem 1(a) of [3] can be extended to the range $p<\frac{n+1}{n-1}$.)

It seems reasonable to conjecture that the result also holds at the endpoint $q=a$, at least when $n>1$. By the above invariance it suffices to consider the case $q=a=2$, so that $p=\frac{2 n}{n-1}, r=\frac{2 n}{n+1}$. Unfortunately the techniques of this paper are not quite powerful enough to resolve this endpoint; if one applies Theorem 10.1 with $B_{0}=L_{x}^{2} L_{\xi}^{2}$ and $B_{1}=L_{x}^{1} L_{\xi}^{\infty}$ one obtains (50) (the required energy and decay estimates are contained in Theorem 2 of [3]), but with the $L_{x}^{p} L_{\xi}^{r}$ norm replaced by that of the real interpolation space

$$
\left(L_{x}^{2} L_{\xi}^{2}, L_{x}^{1} L_{\xi}^{\infty}\right)_{\left(\frac{1}{n}, 2\right)}^{*},
$$

which is between the spaces $L_{x}^{p, 1} L_{\xi}^{r, 1}$ and $L_{x}^{p, \infty} L_{\xi}^{r, \infty}$ but is neither stronger nor weaker than $L_{x}^{p} L_{\xi}^{r}$.

DEPARTMENT OF MATHEMATICS, UCLA, Los ANGELES, CA 90095-1555

Electronic mail: KEEL@MATH.UCLA.EDU

Electronic mail: TAO@MATH.UCLA.EDU

REFERENCES

[1] J. Bergh and J. Löfström, Interpolation Spaces: An Introduction, Springer-Verlag, New York, 1976.

[2] J. Bourgain, Estimates for cone multipliers, Oper. Theory Adv. Appl. 77 (1995), 41-60.

[3] F. Castella and B. Perthame, Estimations de Strichartz pour les èquations de transport cinétique, C. R. Acad. Sci. Paris Sér. I Math. 332 (1996), 535-540.

[4] T. Cazenave and F. B. Weissler, Critical nonlinear Schrödinger equation, Nonlinear Anal. 14 (1990), 807-836.

[5] D. Foschi, Lecture notes for S. Klainerman's graduate course in nonlinear wave equations: Fall 1996, Princeton University, private communication.

[6] J. Ginibre and G. Velo, Smoothing properties and retarded estimates for some dispersive evolution equations, Comm. Math. Phys. 123 (1989), 535-573.

[7] Generalized Strichartz inequalities for the wave equation, J. Funct. Anal. 133 (1995), $50-68$.

[8] L. Hörmander, Estimates for translation invariant operators in $L^{p}$ spaces, Acta Math. 104 (1960), 93-140.

[9] L. Kapitanski, Some generalizations of the Strichartz-Brenner inequality, Leningrad Math. J. 1 (1990), 693-676. 
[10] Weak and yet weaker solutions of semilinear wave equations, Comm. Partial Differential Equations 19 (1994), 1629-1676.

[11] C. E. Kenig, G. Ponce and L. Vega, Oscillatory integrals and regularity of dispersive equations, Indiana Math. J. 40 (1991), 33-69.

[12] S. Klainerman and M. Machedon, Space-time estimates for null forms and the local existence theorem, Comm. Pure Appl. Math. 46 (1993), 1221-1268.

[13] H. Lindblad, A sharp counterexample to local existence of low regularity solutions to nonlinear wave equations, Duke Math J. 72 (1993), 503-539.

[14] H. Lindblad and C. D. Sogge, On existence and scattering with minimal regularity for semilinear wave equations, J. Funct. Anal. 130 (1995), 357-426.

[15] G. Mockenhaupt, A. Seeger and C. D. Sogge, Local smoothing of Fourier integrals and Carleson-Sjolin estimates, J. Amer. Math. Soc. 6 (1993), 65-130.

[16] S. J. Montgomery-Smith, Time decay for the bounded mean oscillation of solutions of the Schrödinger and wave equation, preprint, 1996.

[17] C. Sadosky, Interpolation of Operators and Singular Integrals, Marcel Dekker, New York, 1976.

[18] I. E. Segal, Space-time decay for solutions of wave equations, Adv. Math. 22 (1976), 304-311.

[19] C. D. Sogge, Propogation of singularities and maximal functions in the plane, Invent. Math. 104 (1991), 349-376.

[20] - Fourier Integrals in Classical Analysis, Cambridge University Press, 1993.

[21] Lectures on Nonlinear Wave Equations, International Press, Cambridge, MA, 1995.

[22] E. M. Stein, Singular Integrals and Differentiability Properties of Functions, Princeton University Press, 1970.

[23] W. Strauss, Nonlinear Wave Equations, Regional Conf. Ser. in Math., vol. 73, Amer. Math. Soc., Providence, RI, 1989.

[24] R. S. Strichartz, Restriction of Fourier transform to quadratic surfaces and decay of solutions of wave equations, Duke Math. J. 44 (1977), 705-774.

[25] P. Tomas, A restriction theorem for the Fourier transform, Bull. Amer. Math. Soc. 81 (1975), 477-478.

[26] H. Triebel, Interpolation Theory, Function Spaces, Differential Operators, North-Holland, New York, 1978.

[27] K. Yajima, Existence of solutions for Schrödinger evolution equations, Comm. Math. Phys. 110 (1987), 415-426. 